\title{
Successful Treatment of Iatrogenic Vertebral Artery Pseudoaneurysm with Coil Embolization: Case Report
}

\author{
Chang Wei Zhang ${ }^{1}$, Seidu A Richard ${ }^{1,2,3}$, Chong Wu ${ }^{1}$, Ting Wang ${ }^{1}$, Lun Xing Lui ${ }^{1}$ and Xiao Dong Xie ${ }^{1 *}$ \\ ${ }^{1}$ Department of Neurosurgery, West China Hospital, Sichuan University, Chengdu, Sichuan, PR China \\ ${ }^{2}$ Department of Immunology, Jiangsu University, Zhenjiang, Jiangsu, PR China \\ ${ }^{3}$ Department of Surgery, Volta Regional Hospital, Ghana
}

\begin{abstract}
Introduction: latrogenic vertebral artery injury (IVAI) is a rare intricacy during cervical spine screw fixation or removal surgery. The overall incidence of vertebral artery (VA) injury related to craniocervical surgery is about $0.2 \%$ in literature.

Case presentation: We report a case of 52-year-old male who suffered iatrogenic vertebral artery injury (VAI) as a result of surgical removal of $\mathrm{C} 1-2$ screw that was successfully fixed at posterior cervical spine on account of traumatic atlantoaxial dislocation four (4) years ago. The surgical removal was on going at a periphery hospital when the patient suffered this intricacy with massive bleeding. He was immediately transferred to our facility. CT angiography done at our facility revealed a pseudoaneurysm measuring about $5.5 \mathrm{~cm}$ in diameter at the cervical region.

Conclusions: We successfully treated this pseudoaneurysm with coil embolization without any further neurological deficits. Although the occurrence of iatrogenic VAI pseudoaneurysm as a result of the removal of C1-2 screw is extremely rare, it occurrence can be very fetal with very serious neurological deficits or death.
\end{abstract}

Keywords: Iatrogenic; Pseudoaneurysm; Embolization; Vertebral artery; Angiography

\section{Introduction}

Iatrogenic vertebral artery injury (IVAI) is a rare intricacy after cervical spine screw fixation surgery. The overall incidence of vertebral artery (VA) injury as a result of after cervical spine screw fixation surgery is about $0.2 \%$ that is about $17 / 8213$ operations annually [1]. VA injury can lead to severe blood loss, intradural or extradural hemorrhage, and the development of arteriovenous fistulae or pseudoaneurysms [2-8]. Furthermore, the consequences of VA injure can be fetal and even result in death because of the difficulty in controlling the pulsating hemorrhage which can cause severe hypotension resulting in cardiac arrest and finally death. Therefore, timely diagnosis and intervention of these spontaneous occurrences is crucial in determining the interventional outcome. We represent a case report of iatrogenic vertebral artery pseudoaneurysm, which we successfully managed via urgent endovascular embolization with no neurological deficit. We also discussed the causes for the iatrogenic VAI as well as suitable management options with very minimal or no neurological deficits.

\section{Case Report}

We report a case of 52-year-old male who suffered iatrogenic vertebral artery injury (VAI) as a result of surgical removal of C1-2 screw that was successfully fixed at posterior cervical spine on account of traumatic atlantoaxial dislocation four (4) years ago (Figure 1). The surgical removal was on going at a periphery hospital when the patient suffered this intricacy with massive bleeding and was immediately transferred to our facility. The patient opted for the removal of the screws because of stiffness of his neck. He was not able to flex, extend or rotate his neck one (1) month prior to the surgery. Intraoperatively, while removing of the screw was on going, a sudden, non-pulsatile welling of bright red blood was appeared. Although the blood was adequately tamponaded, the patient remained hemodynamically and neurologically stable during the procedure. CT angiogram done at our facility revealed a pseudoaneurysm arising from the right vertebral artery (V3 segment), just superior to the posterior arch of $\mathrm{C} 1$ while DSA revealed a pseudoaneurysm and extravasation of contrast media in the right vertebral artery at the level of C1-2 (Figure 2). Emergency laboratory investigations done at our facility were all at normal ranges. An emergency interventional operation was carried after his relatives had signed the concern form.

The procedure was performed using $1 \%$ lidocaine as local anesthetic and standard Seldinger technique to access the left femoral artery and placement of 6-French sheath. A $6 \mathrm{~F}$ Envoy catheter was advanced over a 0.035 guidewire and placed at the distal cervical segment of the right vertebral artery. A DSA run showed the pseudoaneurysm arising from the V3 segment. The aneurysm was selected with a 0.014 -inch microcatheter (Enchalon-10, eV3, Plymouth, MN) and a 0.010-inch microguidewire (Transend, Boston Scientific, Natick, MA). Overall, one detachable coil, measuring $15 \mathrm{~mm} \times 30 \mathrm{~mm}$, was inserted into the aneurysm sac. The postprocedural angiogram showed the aneurysm was filled out by the contrast medium (Figure 1). Furthermore, via the left, one detachable coil, measuring $8 \mathrm{~mm} \times 30 \mathrm{~mm}$, was also inserted into the aneurysm sac. The postembolization angiogram showed complete occlusion of the pseudoaneurysm at right vertebral artery

*Corresponding author: Xiao Dong Xie, Department of Neurosurgery, Postgraduate Training Centre, West China Hospital, Sichuan University, 37 Guo Xue Xiang Road, Chengdu, Sichuan, 610041, PR China, Tel: +8618980601975 Fax: +86 28 85422490; E-mail: vipneurology@126.com

Received September 20, 2017; Accepted October 06, 2017; Published October 17, 2017

Citation: Zhang CW, Richard SA, Wu C, Wang T, Lui LX, et al. (2017) Successfu Treatment of latrogenic Vertebral Artery Pseudoaneurysm with Coil Embolization: Case Report. J Vasc Med Surg 5: 342. doi: 10.4172/2329-6925.1000342

Copyright: (c) 2017 Zhang CW, et al. This is an open-access article distributed under the terms of the Creative Commons Attribution License, which permits unrestricted use, distribution, and reproduction in any medium, provided the original author and source are credited. 


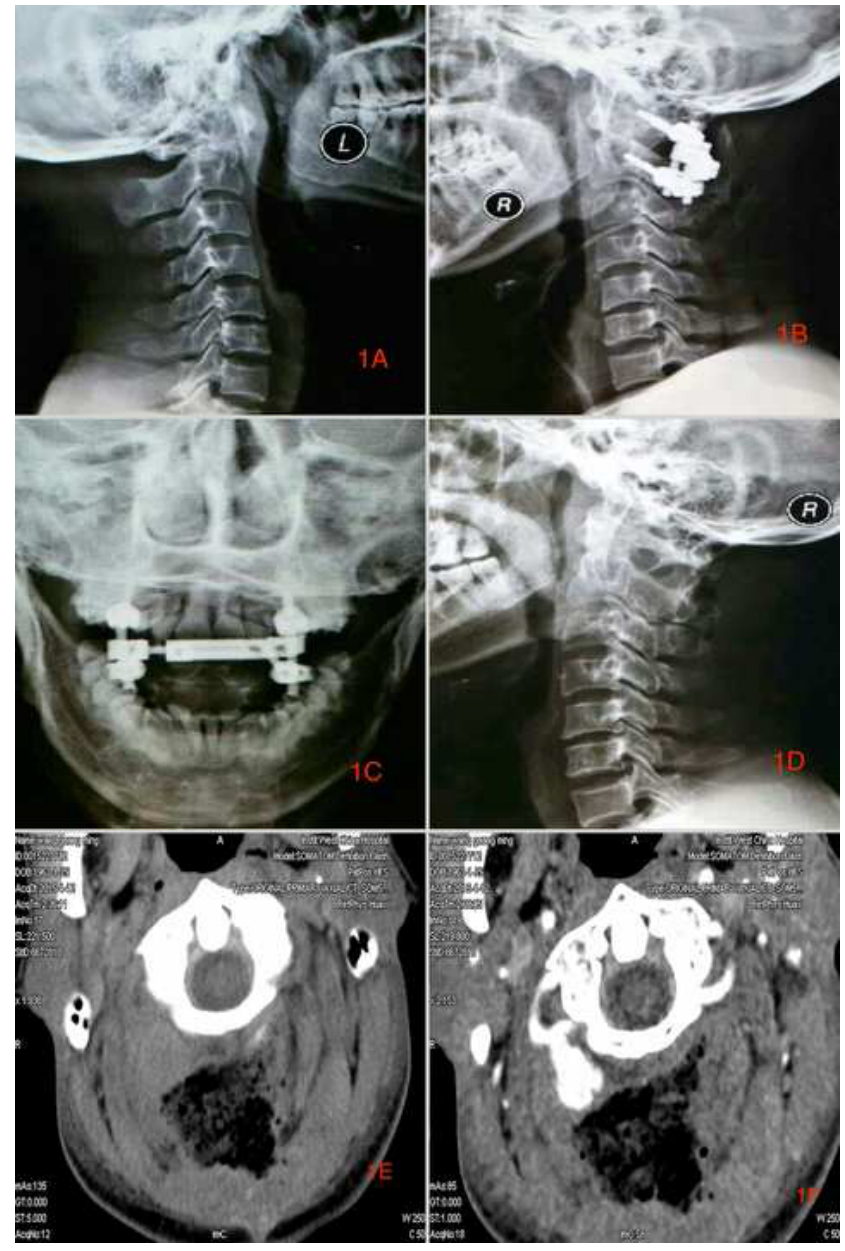

Figure 1: CT-Scan images showing the initial cervical fixation with some fusion of the C1-C2 intervertebral junctions.

(Figure 3). The sheath was removed and a 6-French Angio-Seal is used as a closure device. After the procedure, the patient had an ischemic stroke in the area of the right posterior inferior cerebellar artery (Figure 4). Postoperative evaluation of the patient 3 days after the operation revealed modified Rankin scale (mRS) score of 4 so we discharged him home on the forth $\left(4^{\text {th }}\right)$ day. Out patients' visits were scheduled every three (3) months for one year and later every 6 months. Two (2) years follow-up so far revealed massive improvement of his life.

\section{Discussion}

The vertebral artery consists of four segments. V1 arises from the subclavian or innominate artery, and enters the C6 foramen transversarium. V2 lies within C6-C2 transverse foramina. V3 is from $\mathrm{C} 2$ to the foramen magnum. V4 stretches intradurally from the foramen magnum to basilar artery. If we recognized the normal anatomy and anomalies can avoid vertebral artery injury. We propose that before craniocervical operation, anomalies of the VA should be assessed with CTA/MRA/DSA. Most patients have a dominant VA. The left VA accounts for $50-60 \%$ of the population whereas the right VA accounts for in approximately only $25 \%$. It is said that the remaining $25 \%$ of the population have equivalent vertebral arteries [9]. If the patient has a patent contralateral VA or well-developed collateral circulation from posterior circulation, an injured vertebral artery could be occluded by the neurosurgeon or endovascular team directly. If

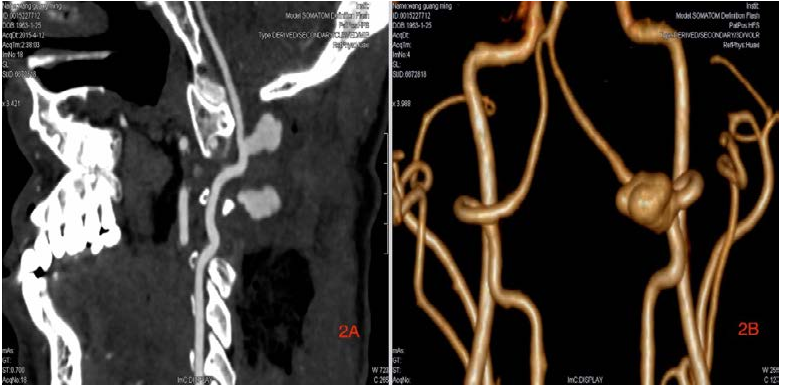

Figure 2: DSA images showing the vertebral artery pseudoaneurysm which occurred during surgery.

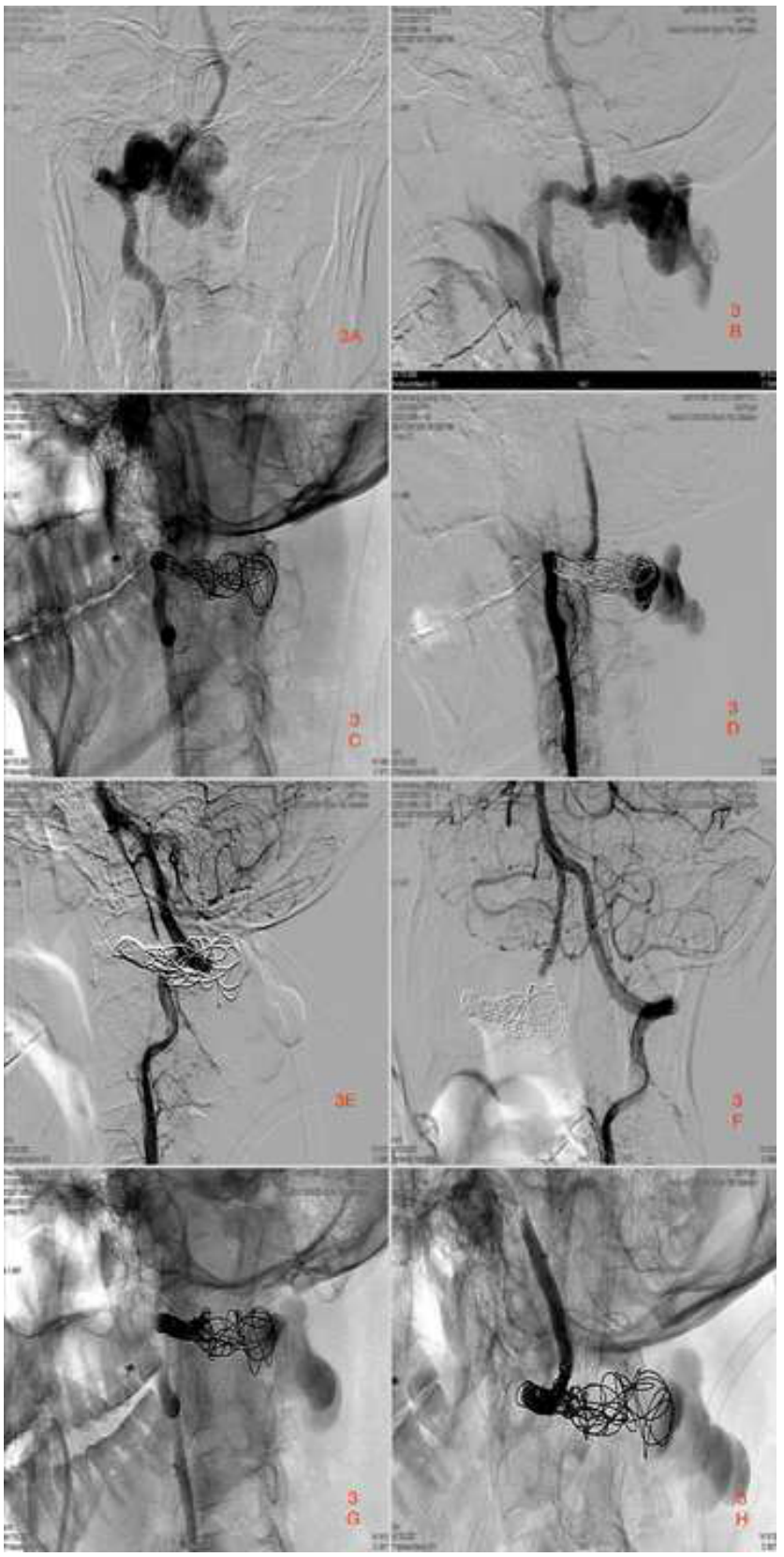

Figure 3: Intraoperative DSA images showing the successfully coiling of the vertebral artery pseudoaneurysm. 

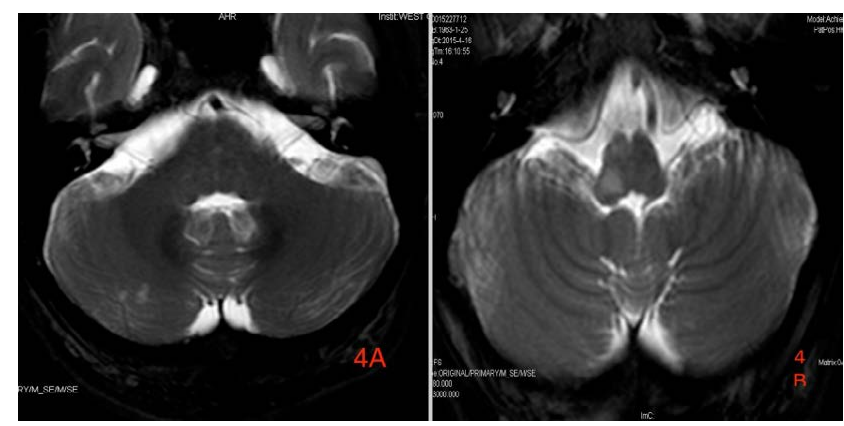

Figure 4: MRI showing an ischemic stroke in the area of the right posterior inferior cerebellar artery.

the contralateral VA is underdeveloped or slender, vessel repair is the preferred treatment modality.

Studies have shown that posterior spine surgeries carry the highest risk of VAI $[6,9]$. There are several ways to prevent intraoperative VA injury. First, careful preoperative evaluation of the dilated or tortuous vertebral artery is most important to prevent iatrogenic VA injury. Failure to recognize an anomalous vertebral artery can result in catastrophic consequences even when lateral decompression is achieved within generally accepted safe limits. Second, an extensive lateral procedure such as decompression of the uncovertebral joints or neural foramen, lateral disc removal should be avoided. Maintaining the midline orientation is essential to adequate decompression of the neural structures, as well as to prevent injury to the vertebral artery. The medial uncovertebral joint should be a guide as to the lateral extent of any dissection or drilling. We speculate the removing of the screw might cause VA injury in this patient. The compression of the screw on the VA prevents hemorrhage.

Once a vertebral artery injury has occurred, the surgeon must always keep the treatment goals in mind in the appropriate order:

- Achieve control of the hemorrhage.

- Prevent acute central nervous system ischemia.

- Prevent postoperative complications such as embolism and pseudoaneurysm.

At first, we should oppresse the parent artery for hemostasis, followed by hemostatic agents such as thrombin soaked gel foam and cottonoids. Sometimes operative treatment for a VA pseudoaneurysm may need proximal ligation of the first or second segments of the VA. However, ligation of the VA distal to the lesion is necessary on account of successive perfusion from distal collaterals $[10,11]$. This therapeutic modality requires exposure of the artery via the foramen transversarium around the injury. If the contralateral/collateral flow is poor, the only remaining option is a bypass surgery. To evaluate this, an aneurysm clip is applied proximal to the injury portion of the vertebral artery while the surgeon looks for significant retrograde flow from the cephalad end [12]. After the bleeding has been controlled, some authors propose close observation and any further treatment given according to the clinical course, whereas others recommend immediate postoperative angiography [10]. In our center, we favor the latter to rule out vascular complications and evaluate adequate collateral circulation to the brain.

Endovascular techniques include detachable balloon, coil, stentassist coil, pipeline and other agents [3,11]. Most patients have a dominant VA. The left VA accounts for $50-60 \%$ of the population whereas the right VA accounts for in approximately only $25 \%$. It is said that the remaining $25 \%$ of the population have equivalent vertebral arteries [3]. If the patient has a patent contralateral VA or sufficient collateral posterior circulation, we could choose direct occlusion of an injured vertebral artery. However, we should preserve posterior inferior cerebellar artery and anterior spinal artery flow in the process. On the other hand, when there is no enough collateral circulation we should ensure that the artery patent. If the contralateral vertebral artery is hypoplastic, vessel repair, with patency of the artery, would be the preferred management via microvascular repair or placement of a stent into the vertebral artery across the injury. In wide-necked pseudoaneurysms, stenting across the neck may result in thrombosis within the sac because of the changes in flow dynamics.

If complete occlusion does not occur, coils can be placed within the lumen of the pseudoaneurysm by passing a microcatheter through the pores of the stent [13]. The micropore flow-diverting stents technique is the most recent improvement in endovascular therapy of intracranial aneurysms. The micropores are designed to obtain a greater change in aneurysm hemodynamics resulting in a diversion of flow within the stent. The SILK (SFD, Balt Extrusion, Montmorency, France) and Pipeline Embolization Device (PED, Covidien Vascular Therapies, Mansfield, MA, USA) promote flow diversion while preserving patency of branch vessels and perforating arteries. Ambekar et al. reported a case of iatrogenic vertebral pseudoaneurysm successfully treated using the PED [11]. In our case, the patient only chooses to sacrifice the parent artery, due to economic reasons. Vertebral artery occlusion or thromboembolic vertebrobasilar events may follow after operation resulting in brain stem or cerebellar dysfunction or may be asymptomatic [14-19].

\section{Conclusion}

Iatrogenic pseudoaneurysms are rare, challenging lesions. If intraoperative profuse bleeding occurs, initial control can be obtained by hemostatic packing. However, due to a risk of delayed bleeding from pseudoaneurysm in such cases, postoperative vertebral angiography should be performed. Considering the high risk of formation of a pseudoaneurysm, prompt recognition is mandatory and endovascular treatment is an important strategy to avoid life-threatening bleeding.

\section{Authors' Contributions}

CWZ conceived the project and designed the study. CWZ, SAR, CW, TW collected and analyzed the patient's data. CWZ and SAR wrote the paper as well as prepared the illustrations. XDX and LXL provided technical assistance in the study. All authors approved the paper for the submission.

\section{Ethics Approval and Consent to Participate}

The ethical committee of the hospital full approved our case study. The patient and his relatives were dually informed about our intension to involve him in a case study and he/they agreed to partake in the study. He/they signed the concern form before the operation was carried out according to all surgical protocols.

\section{Consent for Publication}

The patient and his relatives were dually informed about our intention to publish his case and he/they fully concerted to the use of these documents. The hospital also concerted to the use of this information for publication. 
Citation: Zhang CW, Richard SA, Wu C, Wang T, Lui LX, et al. (2017) Successful Treatment of latrogenic Vertebral Artery Pseudoaneurysm with Coil Embolization: Case Report. J Vasc Med Surg 5: 342. doi: 10.4172/2329-6925.1000342

\section{References}

1. Maughan PH, Ducruet AF, Elhadi AM, Martirosyan NL, Garrett M, et al. (2013) Multimodality management of vertebral artery injury sustained during cervical or craniocervical surgery. Operative Neurosurgery 73: 271-282.

2. Aota Y, Honda A, Uesugi M, Yamashita T, Baba N, et al. (2006) Vertebral artery injury in C-1 lateral mass screw fixation. J Neurosurg: Spine 5: 554-554.

3. Méndez JC, González-Llanos F (2005) Endovascular treatment of a vertebra artery pseudoaneurysm following posterior C1-C2 transarticular screw fixation. Cardiovascular and Interventional Radiology 28: 107-109.

4. Prabhu VC, France JC, Voelker JL, Zoarski GH (2001) Vertebral artery pseudoaneurysm complicating posterior $\mathrm{C} 1-2$ transarticular screw fixation: case report. Surgical Neurology 55: 29-33.

5. Wilson DA, Fusco DJ, Theodore N (2011) Delayed subarachnoid hemorrhage following failed odontoid screw fixation: Case report. Journal of Neurosurgery: Spine 14: 715-718

6. Yilmaz MB, Donmez H, Tonge M, Senol S, Tekiner A (2015) Vertebrojugular Arteriovenous Fistula and Pseudoaneurysm Formation due to Penetrating Vertebral Artery Injury: Case Report and Review of the Literature. Turk Neurosurg 25: 141-145.

7. Lan ZG, Richard SA, Liu J, You C (2017) Chiari type I malformation with cervicothoracic syringomyelia subterfuge as flail arm syndrome. Neurology International.

8. Lan ZG, Richard SA, Lei C, Ju Y (2017) Thoracolumbar Langerhans cell histiocytosis in a toddler. Journal of Pediatric Surgery Case Reports.

9. Choi JW, Lee JK, Moon KS, Kim YS, Kwak HJ, et al. (2006) Endovascular embolization of iatrogenic vertebral artery injury during anterior cervical spine surgery: report of two cases and review of the literature. Spine 31: 891-894.

10. Wright NM, Lauryssen C (1998) Vertebral artery injury in C1-2 transarticular screw fixation: results of a survey of the AANS/CNS section on disorders of the spine and peripheral nerves. Journal of Neurosurgery 88: 634-640.

11. Ambekar S, Sharma M, Smith D, Cuellar H (2014) Successful treatment of iatrogenic vertebral pseudoaneurysm using pipeline embolization device. Case Reports in Vascular Medicine.

12. Devin C, Kang J (2009) Vertebral artery injury in cervical spine surgery. Instructional Course Lectures 58: 717-728.

13. Horowitz MB, Miller G, Meyer Y, Carstens G, Purdy PD (1996) Use of intravascular stents in the treatment of internal carotid and extracranial vertebral artery pseudoaneurysms. American Journal of Neuroradiology 17: 693-696.

14. Friedman D, Flanders A, Thomas C, Millar W (1995) Vertebral artery injury after acute cervical spine trauma: rate of occurrence as detected by MR angiography and assessment of clinical consequences. AJR American Journal of Roentgenology 164: 443-447.

15. Higashida RT, Halbach VV, Tsai FY, Norman D, Pribram H, et al. (1989) Interventional neurovascular treatment of traumatic carotid and vertebral artery lesions: results in 234 cases. American Journal of Roentgenology 153: 577-582.

16. Madawi AA, Casey AT, Solanki GA, Tuite G, Veres R, et al. (1997) Radiological and anatomical evaluation of the atlantoaxial transarticular screw fixation technique. Journal of Neurosurgery 86: 961-968.

17. Prabhu VC, Patil AA, Hellbusch LC, McConnell JR, Leibrock LG (1994) Magnetic resonance angiography in the diagnosis of traumatic vertebrobasilar complications: A report of two cases. Surgical Neurology 42: 245-248.

18. Vinchon M, Assaker R, Leclerc X, Lejeune JP (1995) Vertebrobasilar insufficiency resulting from traumatic atlantoaxial instability: case report. Neurosurgery 36: 839-841.

19. Yee LF, Olcott EW, Knudson MM, Lim RC (1995) Extraluminal, transluminal, and observational treatment for vertebral artery injuries. Journal of Trauma and Acute Care Surgery 39: 480-486. 